Pacific Journal of Mathematics

INDECOMPOSABLE SURFACES IN 4-SPACE 


\title{
INDECOMPOSABLE SURFACES IN 4-SPACE
}

\section{Charles Livingston}

\begin{abstract}
Knotted surfaces in 4-space are constructed which cannot be decomposed as the connected sum of knotted surfaces of lower genus.
\end{abstract}

This paper describes the construction of knotted smooth orientable surfaces in $S^{4}$ which cannot be decomposed as the connected sum of knotted surfaces of lower genus. Examples exist for all genera greater than 0 .

Although it is expected that the knot theory of surfaces in 4-space is much more complicated than that of 2-spheres, examples of this are fairly difficult to construct. The first published example of a knotted torus in $S^{4}$ which is not the connected sum of a knotted sphere with an unknotted torus was constructed by Asano [A]. Subsequently other such examples were constructed by a variety of authors, [BMS], [G], [L], [Lith] and [M].

The constructions of Brunner, Mayland and Simon and of Maeda are of particular interest because they introduced the study of group homology into the study of the knotting of surfaces. (The role of the second homology of the knot group for 2-spheres appeared first in the work of Kervaire [K].) If $F$ is a knotted surface in $S^{4}$ then $H_{2}\left(\pi_{1}\left(S^{4}-F\right)\right)$ is a quotient of $Z^{2 g} \approx H_{2}\left(S^{4}-F\right)$, where $g$ is the genus of $F$. In [BMS] and in [M] examples are constructed in which $F$ is a torus and $H_{2}\left(\pi_{1}\left(S^{4}-F\right)\right)=Z_{2}$. This result was followed up by results of Gordon [G] showing that the $Z_{2}$ could be replaced by any cyclic group. The culmination of this series of results was obtained by Litherland [Lith], who proved that any quotient of $Z^{2 g}$ can occur as the second homology group of the fundamental group of knotted genus $g$ surface in 4-space.

It is fairly clear that none of the results mentioned above can, on their own, produce examples of surfaces with the properties we are aiming for. In fact, in all of these previous works when examples of genus greater than one were constructed they appeared explicitly as connected sums. This paper exploits a combination of the previous 
constructions as well as some detailed group theory calculations to arrive at the desired examples.

To conclude this introduction we will give a brief summary of the techniques that go into the construction. For now we will focus on the genus 2 case. If a knotted surface decomposes as the connected sum of other knotted surfaces then the fundamental group decomposes as the amalgamated free product of other knot groups. The first difficulty encountered is that one of the groups can conceivably be $Z$. This difficulty is resolved by starting with a surface such that the second homology of its group is $Z^{4}$. In this case we will see that if the surface decomposes as a connected sum of tori then each knot group will have second homology $Z^{2}$, and hence cannot be $Z$.

The second difficulty is in showing that the group does not decompose as an amalgamated free product in which each factor has nontrivial second homology. This result depends on a careful analysis of the effect of the various constructions used to build the knotted surface on the structure of the group. It is at this point that some detailed combinatorial group theory also appears.

The construction of examples of genus greater than 2 is only technically more involved than the genus 2 case. Hence the paper will develop the genus 2 example completely. The final section summarizes the changes which are needed to construct higher genus examples.

Before beginning the body of the work, thanks are due to Allan Edmonds for his helpful comments regarding the material.

1. Basic constructions. There are two basic constructions which can be performed on a knotted surface. We will now describe these constructions and describe how they affect the fundamental group of the complement of the knotted surface.

The first of these constructions is a generalization of the spinning construction developed by Artin [Art]. It has been used in many of the constructions of knotted surfaces in 4-space, [A], [L], and [PR].

Let $F$ be a knotted surface in $S^{4}$. As the intersection form of $S^{4}$ is trivial it follows that the normal bundle to $F$ is trivial. Let $s$ denote a section of the normal bundle, viewed as a map of the knotted surface into its complement. The section $s$ is determined uniquely up to homotopy by the restriction that it induce the 0 map on $H_{1}(s(F)) \rightarrow H_{1}\left(S^{4}-F\right)$.

If $\alpha$ is a simple closed curve on $F$, a neighborhood of $\alpha, N$, in $S^{4}$ is diffeomorphic to $S^{1} \times B^{3}$. The pair $(N, N \cap F)$ is diffeomorphic to $\left(S^{1} \times B^{3}, S^{1} \times A\right)$, where $A$ is an unknotted arc in $B^{3}$. A new knotted 


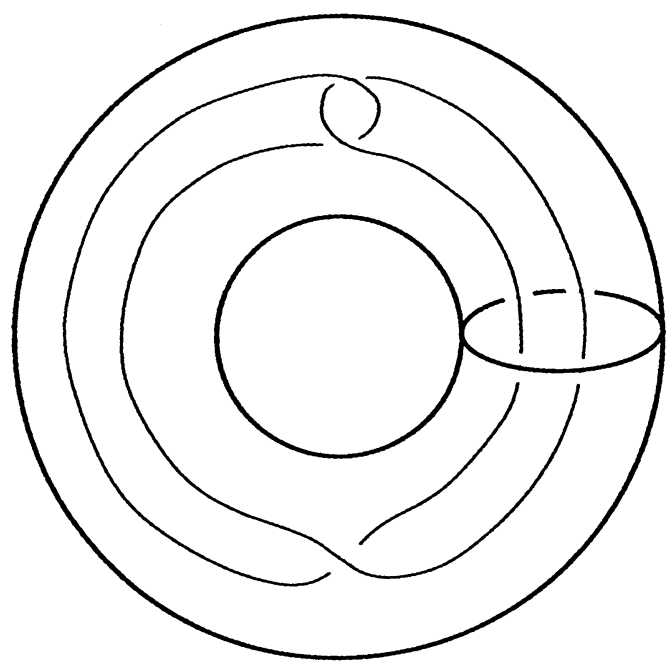

FiguRE 1

surface can be constructed by replacing $S^{1} \times A$ with $S^{1} \times J$, where $J$ is a knotted arc in $B^{3}$. Denote the new surface constructed in this way by $F^{\prime}$.

An argument using Van Kampen's theorem applies to show that $\pi_{1}\left(S^{4}-F^{\prime}\right)$ is the amalgamated free product of $\pi_{1}\left(S^{4}-F\right)$ with $\pi_{1}\left(B^{3}-J\right) \times Z$, where the amalgamation is along a $Z^{2}$. If $s(\alpha)$ is of infinite order in $\pi_{1}\left(S^{4}-F\right)$ then the $Z^{2}$ injects into each factor. In particular, the element $[s(\alpha)]$ is identified with the generator of $Z$ under the amalgamation.

A less obvious observation is that the inclusion map of $H_{2}\left(\pi_{1}\left(S^{4}-F\right)\right)$ into $H_{2}\left(\pi_{1}\left(S^{4}-F^{\prime}\right)\right)$ induced by the inclusion of spaces $S^{4}-N(F) \rightarrow S^{4}-F^{\prime}$ is surjective. This result follows from the observation that the inclusion of $H_{2}\left(S^{4}-N(F)\right)$ into $H_{2}\left(S^{4}-F^{\prime}\right)$ is surjective.

The second construction to be described was discovered by Litherland [Lith]. We will only outline that construction here, isolating the essential results we need to use.

Let $K$ denote the knot in $S^{1} \times B^{2}$ illustrated in Figure 1 and let $T$ denote a knotted torus in $S^{4}$. A regular neighborhood, $N$, of $T$ is diffeomorphic to $\left(S^{1} \times B^{2}\right) \times S^{1}$, and any diffeomorphism of $\left(S^{1} \times B^{2}\right) \times S^{1}$ to $N$ carries $K \times S^{1}$ to a new knotted surface in $S^{4}$. This is Litherland's basic construction. 
In [Lith] it is shown that by iterating this construction, picking the appropriate diffeomorphism at each step, one can construct a knotted torus $T^{\prime}$ for which $H_{2}\left(\pi_{1}\left(S^{4}-T^{\prime}\right)\right)=Z^{2}$. In addition $\pi_{1}\left(S^{4}-T^{\prime}\right)$ is the amalgamated free product of $\pi_{1}\left(S^{1} \times B^{2}-K\right) \times Z$ with $\pi_{1}\left(S^{4}-T^{\prime \prime}\right)$, where $T^{\prime \prime}$ is the torus that results at the next to last stage. The amalgamation is along a $Z^{3}$, and is induced by the splitting of $S^{4}-T^{\prime}$ along $\partial\left(\left(S^{1} \times B^{2}\right) \times S^{1}\right)$. The fact that the $Z^{3}$ can be made to inject into each factor is guaranteed by Lemma 4 of [Lith]. It follows that the section $s$ of the normal bundle of $T^{\prime}$ induces an injection of $\pi_{1}\left(T^{\prime}\right)$ into $\pi_{1}\left(S^{4}-T^{\prime}\right)$.

Note that $H_{2}\left(\pi_{1}\left(S^{1} \times B^{2}-K\right) \times Z\right)=Z^{3}$. This follows from the fact that $H_{2}\left(\left(S^{1} \times B^{2}-K\right) \times S^{1}\right)=Z^{3}$, and that $S^{1} \times B^{2}-K$ is aspherical. In addition we will need the fact that the inclusion of $\pi_{1}\left(S^{1} \times B^{2}-K\right) \times Z$ into $\pi_{1}\left(S^{4}-T^{\prime \prime}\right)$ induces a surjection on the second homology of the groups. This is a consequence of the observation that for any knotted surface $F$, the inclusion of $\mathrm{H}_{2}(N(F)-F)$ into $\mathrm{H}_{2}\left(S^{4}-F\right)$ is surjective.

To summarize the needed observations:

Proposition. There exists a knotted torus $T$ in $S^{4}$ with $\pi_{1}\left(S^{4}-T\right)$ a nontrivial amalgamated free product $(\pi \times Z) * Z^{3} H$, where $\pi=$ $\pi_{1}\left(S^{1} \times B^{2}-K\right)$, and the inclusion of $\pi \times Z$ into $\pi_{1}\left(S^{4}-T\right)$ induces a surjection of $H_{2}(\pi \times Z)$ onto $H_{2}\left(\pi_{1}\left(S^{4}-T\right)\right)=Z^{2}$.

2. The construction of the genus 2 example. In both of the constructions described in the previous sections a 3-dimensional knot group appears. In one case $\pi_{1}\left(B^{3}-J\right)$, in the other $\pi_{1}\left(S^{1} \times B^{2}-K\right)$. In the applications that follow we will need to know that the group does not split in a nontrivial way over $Z$, either as an amalgamated free product or as an HNN construction. Three dimensional techniques allow us to rule out both possibilities. The existence of such a splitting implies the existence of an incompressible, $\partial$-incompressible annulus in the complement of the knot [F]. By ruling out non-prime knots and cabled knots this requirement can be satisfied. In addition, by ruling out a few other possibilities we can assure that the group is not a free product and is not isomorphic to $Z$. The knot in Figure 1 is an example of a knot in $S^{1} \times B^{2}$ with the desired properties. It was used in [Lith]. From now on it will be assumed that the knots used in the constructions satisfy these requirements. 
Let $T$ denote the knotted torus described in the previous proposition, and consider the knotted surface of genus $2, T \# T$. The fundamental group of its complement is given by $G *_{Z} G$, where $G=$ $(\pi \times Z) * Z^{3} H$.

The element $g \in G$ which generates the $Z$ factor of $\pi \times Z$ is of the form $[s(\alpha)]$, where $\alpha$ is a simple closed curve on $T$. Using the inclusion of $G$ into the first factor of $G *_{Z} G$ we get an element, $g_{1}$. Similarly, using the second factor produces an element $g_{2}$. It is important to note that neither $g_{1}$ nor $g_{2}$ is in the amalgamating subgroup. This follows from the observation that both are trivial in $H_{1}\left(S^{4}-T\right)$, while no nontrivial element of the amalgamating subgroup is trivial in $H_{1}$.

The element $g_{1} g_{2}$ is of the form $[s(\gamma)]$ for simple closed curve on $T \# T$. It is possible to apply the first of the constructions described in the previous section along the curve $\gamma$. Denote the resulting surface by $F$, and denote $\pi_{1}\left(S_{4}-F\right)$ by $\Gamma$. This is the desired surface of genus 2.

By the previous remarks we have that

$$
\Gamma=G *_{Z} G *_{Z^{2}}\left(\pi^{*} \times Z\right)
$$

where $\pi^{*}=\pi_{1}\left(B^{3}-J\right)$. Finally, note that the inclusion of $Z^{2}$ into $G *_{Z} G$ is injective.

3. The indecomposability of $F$. The proof that $F$ cannot be decomposed as the connected sum of knotted tori reduces to the following statement concerning $\Gamma$ : if $\Gamma$ decomposes as an amalgamated free product, $A *_{Z} B$ then either $H_{2}(A)=0$ or $H_{2}(B)=0$. The reduction follows immediately from the facts that $H_{2}\left(A *_{Z} B\right)=H_{2}(A) \oplus H_{2}(B)$, that $H_{2}(\Gamma)=Z^{4}$, and that the group of a knotted torus cannot have $H_{2}=Z^{4}$.

The proof of this algebraic statement consists of two steps. In the first it is reduced to a statement not involving group homology. The second step is an argument in combinatorial group theory.

Group homology. Recall first that the inclusion of $\pi \times Z$ into $G$ induces a surjection of $H_{2}(\pi \times Z)$ onto $H_{2}(G)$. Hence the two images of $H_{2}(\pi \times Z)$ in $H_{2}\left(G *_{Z} G\right)$ induced by the natural inclusions will together generate $\mathrm{H}_{2}\left(G *_{Z} G\right)$. Since the inclusion of $G *_{Z} G$ into $\Gamma$ also induces a surjection on $\mathrm{H}_{2}$ it follows that the two images of $H_{2}(\pi \times Z)$ in $H_{2}(\Gamma)$ that arise from the natural inclusions will also generate.

Denote the two inclusions of $\pi \times Z$ into $\Gamma$ by $h_{1}$ and $h_{2}$. Let $f$ denote an isomorphism, $f: \Gamma \rightarrow A *_{Z} B$. If we could show that $f \circ h_{i}(\pi \times Z)$ 
were contained in $A$ for both $i=1$ and 2 we would be done. In this case it would follow that $H_{2}(A)=Z^{4}$, and hence that $H_{2}(B)=0$. Similarly with $A$ and $B$ reversed.

We will show slightly less. In the next step it will be shown that both $f \circ h_{1}(\pi \times Z)$ and $f \circ h_{2}(\pi \times Z)$ are both contained in conjugates of $A$ or both are contained in conjugates of $B$. This suffices to give the desired result, since conjugation of a group induces the trivial action on its homology groups. (See [B], Chapter II, Proposition 6.2.)

Combinatorial group theory. It remains to show that $f \circ h_{1}(\pi \times Z)$ is contained in a conjugate of $A$ or of $B$ and that $f \circ h_{2}(\pi \times Z)$ is contained in a conjugate of the same subgroup. Good references for the combinatorial group theory that follows are [MKS] and [SW]. We begin by quoting directly from [SW].

"We say a group $B$ splits over a subgroup $C$ if $G=$ $A *_{C}$ or $G=A{ }^{*} C B$, with $A \neq C \neq B$. If $G$ splits over some subgroup we say $G$ is splittable. Note that $Z$ is splittable as $Z=\{1\} *\{1\}$.

"Corollary 3.8. If $G=A *_{C}$ or $G=A{ }^{*} C B$ and $H$ is a finitely generated nonsplittable subgroup of $G$, the $H$ lies in a conjugate of $A$ or $B . "$

Note that in the above Corollary it is only necessary to assume that $H$ is nonsplittable over a subgroup isomorphic to a subgroup of $C$.

In our case the amalgamating subgroup is $Z$. Hence the following lemma will apply.

LEMMA 1. If $\pi$ is nonsplittable over $Z$, then $\pi \times Z$ is nonsplittable over $Z$.

Proof. Suppose first that $f: \pi \times Z \rightarrow A *_{Z} B$ is an isomorphism. As $Z \subset \pi \times Z$ is central, $f(Z)$ is contained in the amalgamating subgroup. Since $\pi$ is nonsplittable over $Z$, after conjugation $f(\pi) \subset A$ (or $B$ ). In this case the map $f$ would not be onto.

The second case to consider is with $f: \pi \times Z \rightarrow A * Z$. As above it follows that after conjugation $f(\pi) \subset A$. The argument is completed by noting that unless $f(\pi)$ were contained in the image of $Z$ under the HNN construction it could not be normal. However, $\pi$ is not cyclic.

Assuming that $\Gamma=A *_{Z} B$, apply the above lemma to $\left(\pi^{*} \times Z\right) \subset \Gamma$. It follows that the $Z$ is contained in a conjugate of $A$ or $B$. Suppose 
it is $A$. After conjugating we assume that it is contained in $A$. Denote the generator of $Z$ by $t$. From the construction of the surface it follows that $t=g_{1} g_{2} \in(\pi \times Z) *_{Z}(\pi \times Z) \subset G *_{Z} G$, where $g_{1}$ is the generator of the first $Z$ and $g_{2}$ the second. Applying the lemma above to the $(\pi \times Z)$ factors we see that each is contained in either a conjugate of $A$ or a conjugate of $B$. The proof in the genus two case is completed by showing that both are contained in conjugates of $A$. Here is the necessary lemma.

LEMMA 2. If $g_{1}$ and $g_{2}$ are elements of $A *_{Z} B$ with $g_{1}$ in a conjugate of $A, g_{2}$ in a conjugate of $B$, and $g_{1} g_{2}$ in $A$ it follows that $g_{2}$ is conjugate to an element in the amalgamating subgroup.

Proof. The proof is topological. Let $X$ be a space with $\pi_{1}(X)=A$ and let $Y$ be a space with $\pi_{1}(Y)=B$. The space $W=X \cup S^{1} \times I \cup Y$ has $\pi_{1}(W)=A *_{Z} B$, where the union is formed to correspond to the amalgamation.

There is a map of the twice punctured disk (that is, a closed disk with two open disks in its interior removed) into $W$ with the outer boundary component going to a curve in $X$ representing a homotopy class conjugate to $g_{1} g_{2}$, and with inner boundary curves going to curves in $X$ and $Y$ representing conjugates of $g_{1}$ and $g_{2}$. If that map is made transverse to $S^{1} \times\left\{\frac{1}{2}\right\}$ the preimage of $S^{1} \times\left\{\frac{1}{2}\right\}$ is a collection of circles on the punctured disk. By the injectivity of the amalgamation, it can be arranged that none of these circles bound disks on the punctured disk. (Use an innermost circle argument.) One of the circles must separate the boundary component which is mapped to $Y$ from the other boundary components. The innermost such circle containing the boundary mapped to $Y$ represents an element in the amalgamating subgroup which is conjugate to $g_{2}$.

4. Genus greater than two and concluding remarks. The construction of an example of genus greater than two is essentially the same as the genus two construction. In this case start with the connected sum of $n$ copies of $T$, and apply the second construction along a curve representing an analogous element, $g_{1} g_{2} \cdots g_{n}$. The combinatorial group theory argument that concludes the previous section requires a slightly more careful innermost circle argument and induction. Those details are left to the reader. 


\section{REFERENCES}

[A] K. Asano, A note on surfaces in 4-space, Math. Seminar Notes, Kobe University, 4 (1976), 195-198.

[Art] E. Artin, Zur Isotopie zweidimensionaler Flachen in $R^{4}$, Abh. Mathe. Seminar Univ. Hamburg, 4 (1925), 174-177.

[B] K. Brown, Cohomology of Groups, New York, Springer-Verlag, 1982.

[BMS] A. M. Brunner, E. J. Mayland, and J. Simon, Knot groups in $\mathrm{S}^{4}$ with nontrivial homology, Pacific J. Math., 103 (1982), 315-321.

[F] C. D. Feustel, A generalization of Kneser's conjecture, Pacific J. Math., 46 (1973), 123-130.

[G] C. McA. Gordon, Homology groups of surfaces in the 4-sphere, Math. Proc. Camb. Phil. Soc., 89 (1981), 113-117.

[Lith] R. A. Litherland, The second homology of the group of a knotted surface, Quar. J. Math. Oxford (2), 32 (1981), 425-434.

[L] C. Livingston, Stably irreducible surfaces in $S^{4}$, Pacific J. Math., 116 (1985), 77-84.

[M] T. Maeda, On the groups with Wirtinger presentations, Math. Seminar Notes, Kwansei Gakuin Univ. 1977.

[MKS] W. Magnus, A. Karrass, and D. Solitar, Combinatorial Group Theory, Dover Publications, Inc., New York, 1976.

[PR] T. Price and D. Roseman, Some examples of projective planes and two spheres in 4-space, preprint.

[SW] P. Scott and C. T. C. Wall, Topological methods in group theory, Homological Group Theory, ed. C. T. C. Wall, New York, Cambridge University Press, 1979.

Received March 16, 1987. Supported in part by a grant from the NSF.

INDIANA UNIVERSITY

BLOOMINGTON, IN 47405 


\title{
PACIFIC JOURNAL OF MATHEMATICS EDITORS
}

\author{
V. S. VARADARAJAN \\ (Managing Editor) \\ University of California \\ Los Angeles, CA 90024 \\ HERBERT Clemens \\ University of Utah \\ Salt Lake City, UT 84112 \\ R. FINN \\ Stanford University \\ Stanford, CA 94305
}

\author{
HERMANN FLASCHKA \\ University of Arizona \\ Tucson, AZ 85721
}

Ramesh A. Gangolli University of Washington Seattle, WA 98195

VAUGHAN F. R. JONES University of California Berkeley, CA 94720
ROBION KIRBY

University of California

Berkeley, CA 94720

C. C. MOORE

University of California

Berkeley, CA 94720

HAROLD STARK

University of California, San Diego La Jolla, CA 92093

\section{ASSOCIATE EDITORS}

\author{
R. ARENS \\ E. F. BECKENBACH \\ B. H. NEUMANN \\ F. WOLF \\ K. YOSHIDA \\ (1906-1982)

\section{SUPPORTING INSTITUTIONS}

\section{UNIVERSITY OF ARIZONA} \\ UNIVERSITY OF BRITISH COLUMBIA \\ CALIFORNIA INSTITUTE OF TECHNOLOGY \\ UNIVERSITY OF CALIFORNIA \\ MONTANA STATE UNIVERSITY \\ UNIVERSITY OF NEVADA, RENO \\ NEW MEXICO STATE UNIVERSITY \\ OREGON STATE UNIVERSITY \\ UNIVERSITY OF OREGON \\ UNIVERSITY OF SOUTHERN CALIFORNIA \\ STANFORD UNIVERSITY \\ UNIVERSITY OF HAWAII \\ UNIVERSITY OF TOKYO \\ UNIVERSITY OF UTAH \\ WASHINGTON STATE UNIVERSITY \\ UNIVERSITY OF WASHINGTON
}

The Supporting Institutions listed above contribute to the cost of publication of this Journal, but they are not owners or publishers and have no responsibility for its content or policies.

Mathematical papers intended for publication in the Pacific Journal of Mathematics should be in typed form or offset-reproduced (not dittoed), double spaced with large margins. Please do not use built up fractions in the text of the manuscript. However, you may use them in the displayed equations. Underline Greek letters in red, German in green, and script in blue. The first paragraph must be capable of being used separately as a synopsis of the entire paper. In particular it should contain no bibliographic references. Please propose a heading for the odd numbered pages of less than 35 characters. Manuscripts, in triplicate, may be sent to any one of the editors. Please classify according to the scheme of Math. Reviews, Index to Vol. 39. Supply name and address of author to whom proofs should be sent. All other communications should be addressed to the managing editor, or Elaine Barth, University of California, Los Angeles, California 90024

There are page-charges associated with articles appearing in the Pacific Journal of Mathematics. These charges are expected to be paid by the author's University, Government Agency or Company. If the author or authors do not have access to such Institutional support these charges are waived. Single authors will receive 50 free reprints; joint authors will receive a total of 100 free reprints. Additional copies may be obtained at cost in multiples of 50 .

The Pacific Journal of Mathematics is issued monthly as of January 1966. Regular subscription rate: $\$ 190.00$ a year (5 Vols., 10 issues). Special rate: $\$ 95.00$ a year to individual members of supporting institutions.

Subscriptions, orders for numbers issued in the last three calendar years, and changes of address should be sent to Pacific Journal of Mathematics, P.O. Box 969, Carmel Valley, CA 93924, U.S.A. Old back numbers obtainable from Kraus Periodicals Co., Route 100, Millwood, NY 10546.

The Pacific Journal of Mathematics at P.O. Box 969, Carmel Valley, CA 93924 (ISSN 0030-8730) publishes 5 volumes per year. Application to mail at Second-class postage rates is pending at Carmel Valley, California, and additional mailing offices. Postmaster: send address changes to Pacific Journal of Mathematics, P.O. Box 969, Carmel Valley, CA 93924.

PUBLISHED BY PACIFIC JOURNAL OF MATHEMATICS, A NON-PROFIT CORPORATION Copyright (C) 1988 by Pacific Journal of Mathematics 


\section{Pacific Journal of Mathematics}

\section{Vol. 132, No. $2 \quad$ February, 1988}

Jeffery Marc Bergen and Luisa Carini, A note on derivations with power central values on a Lie ideal ..............................209

Alfonso Castro and Sumalee Unsurangsie, A semilinear wave equation

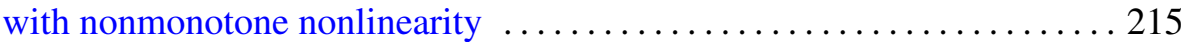

Marius Dadarlat, On homomorphisms of matrix algebras of continuous

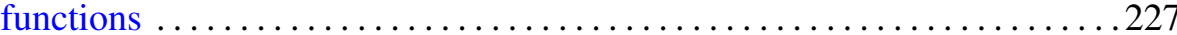

A. Didierjean, Quelques classes de cobordisme non orienté refusant de se

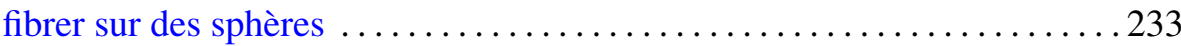

Edward George Effros and Zhong-Jin Ruan, On matricially normed spaces

Sherif El-Helaly and Taqdir Husain, Orthogonal bases are Schauder bases

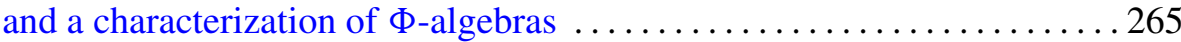

Edward Richard Fadell and Peter N-S Wong, On deforming $G$-maps to be

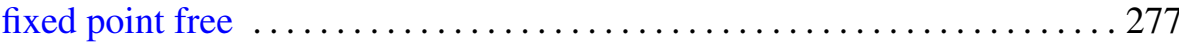

Jean-Jacques Gervais, Stability of unfoldings in the context of equivariant

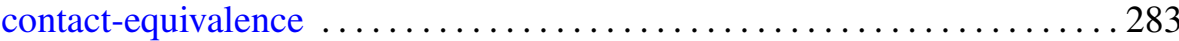

Douglas Martin Grenier, Fundamental domains for the general linear group

Ronald Scott Irving and Brad Shelton, Loewy series and simple projective

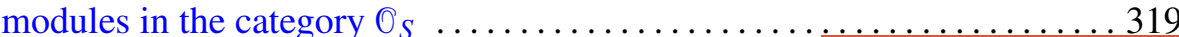

Russell Allan Johnson, On the Sato-Segal-Wilson solutions of the K-dV equation

Thomas Alan Keagy and William F. Ford, Acceleration by subsequence transformations

Min Ho Lee, Mixed cusp forms and holomorphic forms on elliptic varieties

Charles Livingston, Indecomposable surfaces in 4-space 